every three hours, transmitting them by telegraph to the station on the plain. These observations on the summit and those on the plain are compared conjointly with the message which arrives at midday from the Paris Observatory. On these are based the meteorological bulletin of the department.

The station on the plain at Rabanesse is installed in a house provided with a quadrangular tower of 15 metres in height. It is provided with a large shelter for the thermometers, and M. Alluard has had a fine photographic studio constructed, in which he intends to organise a regular service for photographing clouds. Other ingenious and beautiful arrangements have been made here, and the entire establishment, on mountain and plain, is one of the most complete in existence, and may be expected to furnish much valuable meteorological data.

For the illustrations we are indebted to our French contemporary, La Nature.

\section{ON THE APPARATUS EMPLOYED BY THE \\ LATE MR. GRAHAM, F.R.S., IN HIS RESEARCHES ${ }^{1}$}

MR. GRAHAM will probably be best remembered as a chemist, although the most important of his researches where either purely physical, or were devoted to the elucidation of questions which occupy an intermediate position between physics and chemistry. It is specially interesting, therefore, to observe what was the nature of the apparatus he employed in

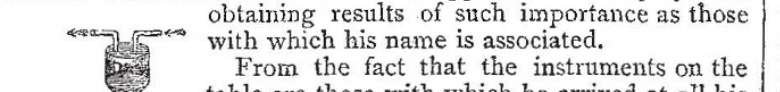
From the fact that the instruments on the table are those with which he arrived at all his more important conclusions, it will at once be evident that the appliances he used were both few and simple. Before I proceed to describe them, I should, as the time at my disposal is very limited, briefly state that Graham's labours were mainly devoted to ascertaining the nature of molecular movement in cases in which he was satisfied that no mass movement could take place, and, as Dr. Angus Smith has pointed out, while Dalton showed the relative weights of the combining quantities, Graham showed the relative magnitude of groups into which they resolve themselves. It is interesting to note that, as Prof. J. P. Cooke has obseryed, while Faraday was so successfully developing the principles of electrical action, Graham, with equal success, was investigating the laws of molecular motion. Each followed with wonderful constancy, as well as skill, a single line of study from first to last, and to this concentration of power their great discoveries are largely due.

Fig. 2. The Royal Society's Catalogue of papers shows that his earliest paper was on the absorption of gases by liquids. It was published in 1826 in Thor son's "Annals of Philosophy"; in it he considers that gases owe their absorption in liquids to their capability of being liquefied, and therefore that solutions of gases in liquids are mixtures of a more volatile with a less volatile liquid. He concludes the paper by saying, that "All that is insisted on in the foregoing sketch is, that when gases appear to be absorbed by liquids they are simply reduced to that liquid inelastic form which otherwise, by cold or pressure, they might be made to assume, and their detention in the absorbing liquid is owing to that mutual affinity between liquids which is so common." It was a theoretical paper only, and no apparatus was even described ; I have quoted it merely because, in his last paper in the Phil. Trans., more than thirty years afterwards, he speaks of the liquefaction of gas in colloids in much the same terms.

In 1829 , the Quarterly Fournal of Science ${ }^{2}$ contains his first paper on the diffusion of gases; he found that the lighter a gas

r Lecture by W. Chandler Roberts, F.R S., Chemist of the Mint, at the Loan Collection, South Kensington.

a Quart. Journ. Sci., ii., $\$ 829$, p. 74 is the more quickly it diffuses away from an open cylinder. The cylinders he employed were nine inches long, and 0.9 inches interior diameter; they were placed in a horizontal position, and the gas under examination was allowed to diffuse outwards through a narrow tube directed either upwards or downwards according as the gas was heavier or lighter than air. It was therefore

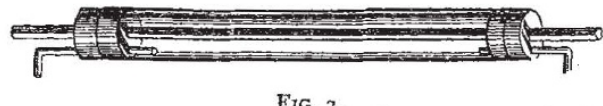

by the aid of a simple cylinder that he was led to believe, as he states in this his first paper, "that the diffusiveness of gases is inversely as some function of their density, apparently the square root of their density." $\mathrm{He}$ subsequently found that so great is the tendency of gases to diffuse into one another, that this mixture or inter-diffusion will take place through apertures of insen-

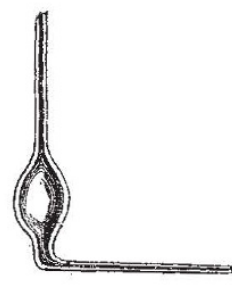

FIG. 4.

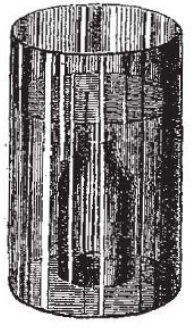

Frg. 5. sible magnitude. And in bis paper in $1834,{ }^{1}$ he treats in detail of diffusion through porous septa, his object being " to establish with numerical exactness the following law of diffusion of gases:-The diffusion or spontaneous intermixture of two gases in contact is effected by an interchange in position of indefinitely minute volumes of the gases, which volumes are not

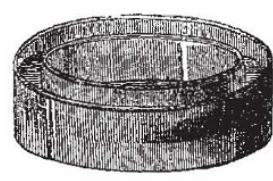

Fig. 6.

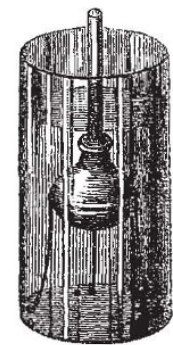

FtG. \%.

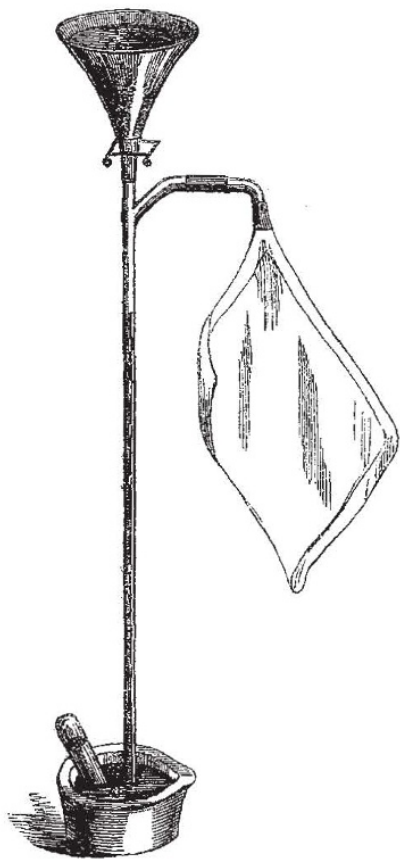

Fig 8 . necessarily of equal magnitude, being, in the case of each gas, inversely proportional to the square-root of the density of that gas." He started from the well-known experiment of Döbereiner, who found, in 1825, that hydrogen kept in; a glass receiver standing over water, escaped by degrees through the fissure into the surrounding air, the water in the receiver rising I Edin, Roy. Soc. Trans., xii., 1834, p. 222. 
to the height of about $2 \frac{3}{4}$ inches above the outer level. In repeating Döbereiner's experiments and varying the circum. stances, Mr. Graham discovered that hydrogen never escaped outwards by the fissure without a certain portion of air penetrating inwards, but with this essential difference, for every volume of air which penetrated into the vessel 3.8 volumes of hydrogen escaped.

The apparatus consisted of a graduated glass tube nearly an inch in diameter, having one end closed by a porous diaphragm of plaster of Paris. This tube was filled with the gas to be examined, and the rise of the mercury indicated the rate at which the interchange of gas and external air took place. He also interposed a bulb two or three inches in diameter between the diaphragm and the graduated tube with a view of increasing the capacity of the instrument, and of avoiding the interference of vapour. In this paper he traced the relation which diffusion bears to the mechanism of respiration, but time will not permit me to consider this question.

These early results were repeated and greatly extended in a paper "On the Molecular Mobility of Gases,"1 but in the experiments there described, thin plates of compressed graphite were principally used. The paper is chiefly remarkable for the clear enunciation of the fact that diffusion is a molecular, and not a mass movement, for Mr. Graham observes : "The pores of artificial graphite appear to be so minute that gas in mass cannot penetrate the plate at all. It seems that molecules only can pass, and they may be supposed to pass wholly unimpeded by friction, for the smallest pores that can be imagined to exist in graphite must be tunnels in magnitude to the ultimate atoms of a gaseous body. The sole motive agency appears to be that intestine movement of molecules which is now generally recognised as an essential property of the gaseous condition of matter.

"According to the physical hypothesis now generally received, a gas is represented as consisting of solid and perfectly elastic spherical particles or atoms, which move in all directions and

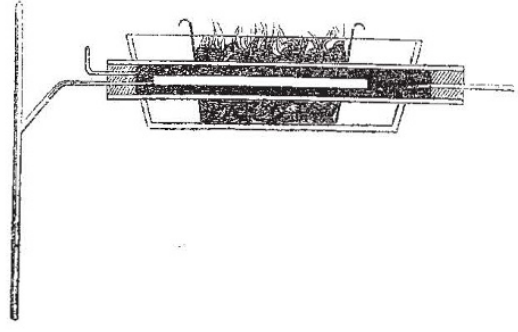

Fig. 9 .

are animated with different degrees of velocity in different gases.". . . . If the vessel containing the gas "be porous like a diffusiometer, then gas is projected through the open channels by the atomic motion described, and escapes. Simultaneousiy, the external air is carried inward in the same manner, and takes the place of the gas which leaves the vessel. To this atomic or molecular movement is due the elastic force, with the power to resist compression possessed by gases."

In order to demonstrate the diffusion of gases it is necessary to exaggerate the conditions of Mr. Graham's experiments. Instead of employing a tube closed with a disc of plaster of Paris, it is better to fix a glass tube into a battery cell and to employ it as the septum through which the gas is diffused. The following experiment was also shown :-A porous battery cell was attached to the short tube of a wash-bottle, both tubes being previousiy turned upwards; when a jar of hydrogen was placed over the battery cell, the gas aiffused through the cell, and the change of pressure caused the water to issue like a fountain several feet in height. I believe this arrangement was devised by Prof. Bloxam.

Now I must ask you to follow me a step further. In 1846 Mr. Graham read a paper before the Royal Society "On the Motion of Gases." He showed that the effusion of gases through a minute hole in a platinum plug left no doubt of the truth of a general law that different gases pass through minute apertures in times which are as the square roots of their respective specific gravities, or with velocities which are inversely as the square roots of their specific gravities; or in other words, he experimentally verified the mechanical law that the velocity with which a gas rushes into a vacuum through such an aperture, is the same as that which a heavy body would acquire in falling from the height of an atmosphere, composed of the gas in question, of uniform density throughout. The relative rates of effusion and diffusion are alike, but Mr. Graham is careful to observe that the phenomena are essentially different in their nature. The former affects masses of gas, the latter (diffusion) only affects molecules.

The apparatus Mr. Graham employed consisted of two glass jars; the one containing the gas to be examined was placed in a pneumatic trough, and the other stood on the plate of an airpump. They were in connection, a series of tubes containing the usual reagents for purifying and drying the gas being interposed between them. The jar on the air-pump was exhausted, and the gas entered it through a minute orifice in a platinum disc, the rate of passage being observed by the aid of a mercurial column.

Three years later Mr. Graham published a papar giving the results of an investigation on what he considered to be a funda. mental property of the gaseous form of matter, which he termed transpiration. He employed capillary tubes, and found that effusion and transpiration differed widely $;{ }^{1}$ " for if the length of the tube is progressively increased, and the passage for all gases becomes greatly slower, the velocities of the different gases are found to diverge rapidly from their effusion rates." The veloci. ties at last, however, attain a particular ratio with a given length of tube and resistance, and preserve the same relation to each other for greater lengths and resistances, the most simple result probably being that of hydrogen, which has exactly double the transpiration rate of nitrogen, the relation of these gases as to density being as 1 : 14 .

\begin{tabular}{|c|c|c|c|c|}
\hline & $\begin{array}{l}\text { Diffusion } \\
\text { Velocities. }\end{array}$ & $\begin{array}{l}\text { Effusion } \\
\text { Velocities. }\end{array}$ & $\begin{array}{c}\text { Transpi:ation } \\
\text { Velocities. }\end{array}$ & $\begin{array}{l}\text { Rates of Pas- } \\
\text { sage through } \\
\text { Caoutchouc. }\end{array}$ \\
\hline 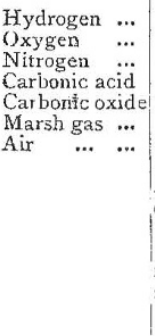 & \begin{tabular}{|c|}
3.83 \\
0.9487 \\
1.0143 \\
0.812 \\
1.0149 \\
$r .344$ \\
$1 \cdot 0$ \\
About 9600 c.c. \\
of air pass per \\
minute \\
through r sq. \\
metre of stucco \\
2.5 mm. thick.
\end{tabular} & $\begin{array}{c}3.613 \\
0.950 \\
10164 \\
0.821 \\
I .0123 \\
I .322 \\
I 0 \\
\\
78.3 \mathrm{cc} \text { c. of air } \\
\text { pass per minute } \\
\text { through a cer- } \\
\text { rain smallaper- } \\
\text { ture in a brass } \\
\text { plate. }\end{array}$ & \begin{tabular}{|c|}
2066 \\
0.903 \\
$r .030$ \\
1.237 \\
1.034 \\
1.639 \\
$r^{\circ} \circ$ \\
62.9 c.c. of air \\
pass perminute \\
through a glass \\
cube 6.6 metres \\
long and \\
$0.55 \mathrm{~mm}$ in \\
diameter.
\end{tabular} & $\begin{array}{c}473 \\
2.224 \\
0.870 \\
11.8 \times 9 \\
0.968 \\
1869 \\
10 \\
\text { 16.9 c.c. of air } \\
\text { pass perminute } \\
\text { through one } \\
\text { square metre of } \\
\text { caoutchouc } \\
0.02 \text { mm. thick. }\end{array}$ \\
\hline
\end{tabular}

Note- - It is impossible to make the four columns strictly comparable on account of the difference of the conditions under which the experiments were made.

Thus, in what are very nearly Mr. Graham's own words, a gas may pass into a vacuum in three different modes; that is, by effusion, transpiration, or diffusion, and I hope you will bear with me while I recapitulate them.

I. The gas may enter the vacuum by effusion, that is, by passing through a minute aperture in a thin plate, such as a puncture in platinum-foil made by a fine steel point. The rela. tive times of the effusion of gases in mass are similar to those of the molecular diffusion, but a gas is usually carried by the former kind of impulse with a velocity many thousand times as great as is demonstrable by the latter.

2. If the aperture of efflux becomes a tube, the effusion rates are disturbed. The rates of flow of different gases, however, assume again a constant ratio to each other when the capillary tube is so elongated that the length exceeds the diameter by at least 4,000 times. The transpiration rates appear to be independent of the material of the capillary ; they are not governed by specific gravity, and are indeed singularly unlike the rates of effusion. The ratios appear to be in direct relation with no other known property of the same gases, and they form a class of phenomena remarkably isolated from all that is at present known of gases.

For instance it will be seen by the table already given that the rate of carbonic acid which is low for effusion and diffusion, becomes comparatively rapid when the gas passes by transpirâtion.

3. A plate of compressed graphite, although it appears I Phil. Trans., 1849 , p. 349 
to be practically impermeable to gas by either of the two modes of passage just described, is readily penetrated by the agency of the molecular or diffusive movement. The times of passage through a graphite plate into a vacuum have no relation to the capillary transpiration times of the gases, but they show a close relation to the square roots of the densities of the respective gases, and agree with the theoretical times of diffusion usually ascribed to the same gases.

These latter results were obtained by the graphite diffusiometer of which a sketch is given (Fig. I). It stood over mercury, and was raised or lowered by an arrangement introduced by Prof. Bunsen.

Mr. Graham subsequently employed the barometrical diffusiometer shown in Fig. 2. It consists of a tube in which a Torricellian vacuum could be produced. The upper end was closed by the porous septum, and a slow stream of the gas under examination was allowed to pass over the plate through the indiarubber hood by which it was covered.

I might mention that the very exact and illustrious experimenter, Prof. Bunsen, was led to doubt the accuracy of Graham's law of the diffusion of gases, but he employed plugs of plaster of Paris which impaired the results by introducing the phenomenon of transpiration; and probably also as $\mathrm{Mr}$. Graham observed to me, by an actual retention of hydrogen in the pores of the plaster It is interesting from our point of view, because it shows that the simple apparatus employed by Mr. Graham really gave the only trustworthy results.

The results of the later experiments led him to prove that mixed gases might be separated from each other by diffusion. Stems of tobacco-pipes were employed, arranged inside a glass tube, which could be rendered vacuous, the mixed gases being passed through the tobacco-pipe. For example, when this explosive mixture of 66 per cent. of hydrogen, and 33 per cent. of oxygen is passed through this tube (Fig. 3) a mixture is obtained containing only 9.3 per cent. hydrogen, and is therefore nonexplosive. With air it was found possible to concentrate the oxygen by 3.5 per cent.

With the apparatus now before us (Fig. 4) Mr. Graham subsequently worked on liquid transpiration in relation to chemical composition. He started from the discovery of $\mathrm{M}$. Poiseuilie, that a definite hydrate of one equivalent of alcohol with six equivalents of water is more retarded than alcohol, containing either a greater or a smaller proportion of water. The rate of transpiration depending upon chemical composition and affording an indication of it, it thus appeared probable that a new physical property might become available for the determination of the chemical constitution of substances, and the experiments appeared to establish "the existence of a relation between the transpirability of liquids and their chemical composition. It is a relation analogous in character to that subsisting between the boiling point and composition so well defined by Hermann Kopp." 1 The apparatus consists of a strong glass jar closed at the top by a brass plate into which a condensing syringe is screwed. This plate also had a tube screwed into it, and into the tube the glass bulb with a long capillary tube was fixed. The fluid under examination was placed in the bulb, which communicated freely with the interior of the jar, containing compressed air.

To revert to the chronological order. His next paper in December, 1849 , formed the Bakerian lecture of the Royal Society. It was on the Difusion of liquids, and the only apparatus employed was very similar to that adopted in his earliest paper on the diffusion of gases; it consisted of a bottle and glass jar (Fig. 5), the fluid under examination being placed in the bottle, which was immersed in the water with which the jar was filled. With this simple apparatus he found that when two liquids of different densities, and capable of mixing, are placed in contact, diffusion takes place between them much in the same manner as between gases, except that the rate of diffusion, which varies with the nature of the liquids, the temperature and the degree of concentration is slower. Common salt when placed in the inner vessel will diffuse twice as rapidly as sulphate of magnesia, and this salt will diffuse twice as rapidly as gum arabic. Subsequently Mr. Graham modified the disposilion of the apparatus and simply introduced the salt to be diffused by means of a pipette to the bottom of a jar filled with water. These experiments led to the very remarkable and important discovery that different compounds might be separated from each other by diffusion, and this was not all, for it was proved that a partial decomposition of chemical compounds was effected by diffasion. Thus ordinary alum was partially decomposed into sulphate of potassium and r Phil. Trans., I86r, p. 373 . sulphate of aluminium, which is less diffusible than the firstnamed salt. Mr. Graham considered this research to be very important, and he remarks, "in liquid diffusion we appear to deal no longer with chemical equivalents or Daltonian atoms, but with masses even more simply related to each other in weight." We may suppose that the chemical atoms "can group together in weights which appear to have a simple relation to each other. It is this new class of molecules which appear to play a part in solubility and liquid diffusion, and not the atoms of chemical combination."

Continuing the investigation he described in a paper of singular beauty, his well-known experiments on the varying rates of liquid diffusion of various soluble substances, which led him to divide them into crystalloids and colloids, the former having a rapid diffusion rate, the latter being marked by low diffusibility. He placed the substance under experiment in a tambourine of parch. ment paper (Fig. 6) which was floated on the surface of a comparatively large volume of water, the highly diffusive crystalloid passed through the membrane, the colloid remained behind, for "the diffusion of a crystalloid appears to proceed even through a firm jelly with little or no abatement of velocity."

I have here the very interesting series of colloids prepared by Mr. Graham, and of these perhaps the most interesting is the soluble silicic acid. If silicate of soda is poured into diluted hydrochloric acid, the acid being maintained in large excess, a solution of silicic acid is obtained. But this solution also contains, in addition to the silicic acid, chloride of sodium, from which it may be freed by the action of dialysis, and by this means a solution, which is not in the least viscous, is obtained, containing I4 per cent of silicic acid. The coagulation of the silicic acid is effected, however, by the addition of a solution containing the $\frac{1}{1}$ th part of any alkaline or earthy carbonate. Mr. Graham therefore described this gelatinous state as the "pectous," as distinguished from the "peptous" or dissolved form.

By a similar process Mr. Graham obtained specimens of soluble alumina, peroxide of iron, chromic oxide, and stannic acid, all of which have their pectous and peptous states. And he showed that in most cases alcohol, sulphuric acid, and glycerine can replace part of the water of these colloids. I cannot describe these interesting substances now, nor can $I$ do more than remind you of the use of dialysis in medico-legal inquiries. I must content myself with summing up a few of Mr. Graham's conclusions with reference to crystalloids and colloids. Although chemically inert, in the ordinary sense, colloids possess a compensating activity of their own, arising out of their physical properties. While the rigidity of the crystalline structure shuts out external impressions, the softness of the gelatinous colloid partakes of fluidity, and enables the colloid to become a medium for liquid diffusion like water itself. Another and eminently characteristic quality of colloids is their mutability, as fluid colloids often pass from the fluid to the pectous or gelatinous condition under the slightest influences. The colloid is, in fact, the dynamic state of matter, the crystalloid being the statical condition. The colloid possesses eneroy, and it may be looked upon as the primary source of the force appearing in the phenomena of vitality."

The next instruments to be considered are those with which $\mathrm{Mr}$. Graham studied osmotic force. When a solution of a salt, or a liquid, is separated by a membrane or porous diaphragm from a mass of water, a flow of liquid takes place from one side of the septum to the other. This action was discovered by Dutrochet and is known as osmose. Dutrochet and Mr. Graham both used a narrow glass tube, having a funnel-shaped expansion at the bottom, covered at that end by a plece of bladder (Fig. 7). $\mathrm{Mr}$. Graham also used porous earthenware and albuminated calico.

In some cases the flow of liquid into the bulb is sufficiently powerful to sustain a column of water many inches high in the glass tube. Dutrochet inferred from his experiments that the velocity of the osmotic current is proportional to the quantity of salt or other substance originally contained in the solution. He attributed the action of the septum to capillarity, but Mr. Grakam ulitimately considered that the water movement in osmose is " an affair of hydration and dehydration of the substance of the membrane or other colloid septum," and that the diffusion of a saline solution only acts by affecting the hydration of the septum. The outer surface of the membrane being in contact with pure water, tends to hydrate itself in a higher degree than the inner surface does, the latter surface being sup. posed to be in contact with a saline solution. When the full 
hydration of the outer surface extends through the thickness of the membrane and reaches the inner surface, it there receives a check. . . The contact of the saline fluid is thus attended by a continuous catalysis of the gelatinous hydrate, by which it is resolved into a lower gelatinous hydrate and free water. Now this question of hydration is perhaps the most remarkable instance of the persistent continuity of Mr. Graliam's work, as Dr. Odling has pointed out, ${ }^{1-}$ "it is noteworthy that for him (Mr. Graham) osmosis became a mechanical effect of the hydration of the septum; that the interest attaching to liquid transpiration was the alteration in rate of passage conseguent on an altered hydration of the liquid, and that the dialytic difference between crystalloids and colloids depended on the dehydration of the dialytic membrane of the former class of bodies only."

I must now direct your attention to a section of Mr. Grabam's work, which, although it was the last, was a reversion to some of his very earliest experiments. In 1829 , under the tille, "Notice of the Singular Inflation of a Bladder," he described ti:e following experiment:-A. bladder two-thirds filled with carbonic acid was introduced into a bell jar filled with carbonic acid gas; atter the lapse of some hours the bladder was found to contain 35 per cent. of carbonic acid, and to have become distended. Mr. Graham observes :- "M. Dutrochet will probably view in these experiments the discovery of endosmose acting upon aexiform matter as he observed it to act on bodies in a liquid state. Unaware of the speculations of that philosopher at the time the experiment was made, I fabricated the following theory to account for them :-The jar of carbonic acid standing over water, the bladder was moist, and we know it to be porous. Between the air in the bladder and the carbonic acid without there existed capillary canals through the substance of the blad. der, filled with water. The surface of the water at the outer extremities of these canals being exposed to carbonic acid, a gas soluble in water would necessarily absorb it. But the gas in solution . . . permeated the canal, and passed into the blad. der and expanded it." 2

Xou will remember that in the concluding experiments on the diffusion of gases Mr. Graham employed a tube, closed with a. graphite disc (Fig. 2), in which a Torricellian vacuum could be produced. In his experiments on the peneiration of different gases through membranes the same apparatus was employed, only the disc of graphite was replaced by a film of india-ribber. He found that gases penetrated to the vacuous space at the rates given in the last column of the table (p. 512). You will observe that the gas which penetrates most rapidly is carbonic acid, and you will also see that the rates of passage are in no way connected either with those of diffusion or transpiration.

A comparison of the relative rates of passage of oxygen and nitrogen led to a most remarkable experiment. Oxygen penetrates $2 \frac{1}{2}$ times as fast as nitrogen, therefore by dialysing air Mr. Graham actually increased the quantity of oxygen from $20^{\prime} 8$ to $4 \mathrm{I}$ per cent., just as he had effected, by the aid of a tobacco-pipe, a partial separation of oxygen from air by the slightly greater diffusion velocity of nitrogen. The Torricellian vacuum was ill adapted for the experiments, and $\mathrm{Mr}$. Graham gladly availed himself of the mercurial exhauster devised by Dr. Hermann Sprengel, and he considered that without the aid of this instrument it would have been impossible to conduct certain portions of the research. He was thus able to use larger septa of india-rubber, bags of waterproof silk being found to be most convenient (Fig. 8). The vacuum was not even absolutely necessary, for the penetration of the nitrogen and oxygen of air through rubber into a space containing carbonic acid could be readily effected, the gas being absorbed by potash at a certain stage of the operations.

Mr. Graham considered this penetration to be due to an actual dissolution of the gas in the substance of the india-rubber, for, as he observes, "gases undergo liquefaction when absorbed by liquids and by soft colloids like india-rubber," words I think of interest, when we remember that the sentence only marks a slight extension of the view he expressed in his first paper in 1829 .

These discoveries led $\mathrm{Mr}$. Graham to inquire whether it was probable that the discovery of MM. Troost and Deville of the penetration of red-hot platinum and iron tubes by hydrogen, could be due to an actual absorption and liquefaction of the gas in the pores of the metal, and by submitting the question to the test of experiment it was proved that such an absorption did take place.

I Lecture on "Prof. Graham's Scientific Work," Royal Institution, January, 1870.
Quart. Journ. Sci., $x 829$, p. 88.
For instance, palladium was found to act as platınun only in a more marked manner. A tube of palladium when attached to the mercurial exhauster did not allow hydrogen to pass in the cold, but when heated to redness in an atmosphere of hydrogen the gas passed through the walls of the tube at the rate of 4,000 cubic centimetres per square metre in an hour (Fig. 9). This led to the remarkable discovery of the absorption or occlusion of gases by metals. It was found that nearly all metals appear to select one or more gases. Silver, for instance, absorbs many times its volume of oxygen, and under certain circumstances gives it out again on cooling. Iron is specialiy characterised by its absorp. tion of carbonic oxide, but it also retains hydrogen, and this fact led Mr. Graham to extract from meteoric iron, the gas that probably affected its reduction to the metallic state, and which certainly exists in the atmosphere of certain stars.

The most remarkable results were obtained with palladium. I called your attention at the beginning of the lecture to the index which you will observe has moved six inches.

I will now describe the apparatus; it consists of a tall jar filled with acidulated water; at the bottom of the jar two wires are fixed, and these wires are parallel throughout the entire length of the jar. Each is attached to the short arm of a lever, the longer arms of which are about five feet long. One wire is of palladium, the other of platinum, and they form the electrocles of a smail battery capable of decomposing the water. The palladium now forms the negative electrode, and is freely absorbing hydrogen, the excess of which is escaping from its surface. The absorption of hydrogen has been attended by a considerable expansion, as, is shown by the fall of the index. The index attached to the platinum wire has of couse remained stationary.

This expansion enabled Mr. Groham to calculate the censity of the gas in its cendened form, and for reasons which I cannot give you now he was led to believe that hydrogert gas is the vapour of a white magnetic metal of specific gravity $0^{\circ} 7$.

Now by taking palladium which has been charged in the manner you have seen, and heating it invacuo, I can actually extract and show you the hydrogen it contained. This little medal of palladium contains an amount of gas condensed into it which would be equivalent to a column of gas more than 2 yard high, and of the diameter of the medal.

The story of Mr. Graham's work has been much better told by Odling, Williamson, Hofmann, and Angus Smith, but what does it teach us from a point of view of a collection of scientific apparatus? Surely that, aithough in certain researches or for accurate observation and measurement, delicate and complicated instruments may be necessary, the simplest appliances in the hands of a man of genius may. give the most important results. Thus we have seen that with a glass tube and plug of plaster of Pais, Mr. Graham discovered and verified the law of diffusion of gases. With a tobacco-pipe he proved indisputably that air is a mechanical mixture of its constituent gases. With a tam. bourine and a basin of water he divided bodies into crystalloids and colloids; and obtained rock crystal and red oxide of iron soluble in water. With a child's indiarubber balloon filled with carbonic acid he separated oxygen from atmospheric air, and established points, the importance of which, from a physiological point of view, it is impossible to overtate. And finally, by the expansion of a palladium wire, he did much to prove that hydrogen is a white metal.

\section{GERMAN EXPEDITION TO SIBERIAI}

"WE stayed in Iepsa until May 17. We obtained some varieties of lizards, one kind of frog, and a toad, a kind of fish like the barbel, and all sorts of varieties of cobitis, but: no salmon. We obtained only a few beetles and butterfies, but we had a rich collection of the flora. On May $\mathrm{I}_{3}$ and $\mathrm{I}_{4}$ we made a short excursion into the mountaius and found several new kinds of birds differing decidedly from the European kinds, e.g., the Cinclus leucogaster, with the white belly, the Motacilla personata, the Pica leucoptera, a fine Carduelis, and a splendid specimen of the red-finch.

"On May $\times 5$ we made a long excursion to the Dschasyl Kul (green lake), 6,000 feet above the level of the sea. The abundance of trees and bushes has a most agreeable effect, and above all is the mild red and pink of the wild apple-tree (Piras Sieve rianus) pleasing to the eye. The lake, lying amongst high

1 The second letter dates from Saissan, in Russian Turkestan, May 27, 2876. Continued from p. 359. 